\title{
Cardiac iron overload promotes cardiac injury in patients with severe COVID-19
}

\author{
Maria J. Baier ${ }^{1} \cdot$ Stefan Wagner ${ }^{1}$. Julian Hupf ${ }^{2} \cdot$ Katja Evert $^{3} \cdot$ Matthias Evert $^{3} \cdot$ Samuel Sossalla $^{1}$. \\ Carsten Jungbauer ${ }^{1} \cdot$ Lars S. Maier $^{1} \cdot$ Stefan Neef ${ }^{1} \cdot$ Julian Mustroph $^{1}$ (I)
}

Received: 24 June 2021 / Accepted: 13 October 2021 / Published online: 20 October 2021

(c) The Author(s) 2021

During the current SARS-CoV-2-pandemic, involvement of multiple organs in addition to the respiratory tract has been observed. Cardiac manifestations, such as acute heart failure, arrhythmias or acute coronary syndrome, are known complications in severe disease courses [1].

Multiple studies have reported hyperferritinemia in patients with COVID-19 infection and suggested dysregulated iron metabolism in severe COVID-19 disease courses [2]. Recently, iron deficiency was linked to significantly longer hospital stay and the need for ICU admission and mechanical ventilation $[3,4]$. However, no direct correlation between cardiac iron homeostasis and severity of COVID-19 infection has been shown yet.

Dysregulated iron homeostasis is accompanied by ferroptosis, a newly identified type of controlled cell death, which is triggered by intracellular iron accumulation, decreased iron storage capacity and glutathione depletion, leading to excessive reactive oxygen species (ROS) formation, lipid peroxidation and, consequently, cell death [5].

In this study, we investigated iron metabolism in left ventricular myocardium of patients who had died due to severe COVID-19 and assessed cardiac iron content as well as ferroptosis pathways in these patients. To our knowledge, this is the first study showing a direct relationship between COVID-19 infection, cardiac iron overload, and cardiac injury in the myocardium.

Julian Mustroph

julian.mustroph@ukr.de

1 Department of Internal Medicine II (Cardiology), University Heart Center Regensburg, University Hospital Regensburg, Franz-Josef-Strauß-Allee 11, 93053 Regensburg, Germany

2 Emergency Department, University Hospital Regensburg, Franz-Josef-Strauß-Allee 11, 93053 Regensburg, Germany

3 Institute of Pathology, University of Regensburg, Universitätsstraße 31, 93053 Regensburg, Germany
All investigations conform to the Declaration of Helsinki. Informed consent for autopsies involving scientific investigations had been obtained from relatives of all deceased patients, which was performed in accordance with institutional and ethics guidelines. We obtained left ventricular cardiac tissue from autopsies of patients who had died of COVID-19. In all these patients, infection was verified by PCR from respiratory material, and all patients had symptomatic disease, which was determined to be the cause of death. Because control tissue is extremely rare, we used a combination of left ventricular tissue from patients whose hearts were intended for heart donation that ultimately could not be performed ("NF" = non-failing, for iron assay and qPCR), and from patients who had died from sepsis or other respiratory infections ("SEPSIS", for qPCR and immunofluorescence). Clinical characteristics of the "COVID-19" and "SEPSIS" patient groups can be found in Table 1. No clinical data is available regarding the non-failing group.

We assessed the iron content in left ventricular myocardium using an iron assay kit (ab83366, abcam), which was performed according to manufacturer's instructions. Briefly, $10 \mathrm{mg}$ of left ventricular tissue was homogenized in $500 \mu \mathrm{l}$ Iron Assay Buffer $(+10 \%$ complete protease inhibitor cocktail, Roche diagnostics). After centrifugation $(16,000 \times g$, $10 \mathrm{~min}$ ) the supernatant was collected and incubated with Iron Reducer $\left(37^{\circ} \mathrm{C}, 30 \mathrm{~min}\right)$ to assess total iron. Afterwards, $100 \mu \mathrm{l}$ of standard $(0,2,4,6,8,10 \mathrm{nmol} /$ well $)$ and sample probes (in duplicates) were incubated with $100 \mu \mathrm{l}$ Iron Probe for $60 \mathrm{~min}$ at $37^{\circ} \mathrm{C}$. Absorption was measured at $593 \mathrm{~nm}$. Total iron concentration in the samples was calculated using standard curve and sample volume.

Western Blot analysis was performed as described previously [6]. Briefly, LV myocardium was mechanically homogenized (using a stainless steel pestle) in Tris buffer containing (in mmol/L): 50 Tris- $\mathrm{HCl}, 200 \mathrm{NaCl}$, $20 \mathrm{NaF}, 1 \mathrm{Na}_{3} \mathrm{VO}_{4}, 1$ DTT [pH 7.4] and protease inhibitor cocktail. Protein concentration was determined by BCA 
Table 1 Baseline characteristics of CTRL and COVID-19 patients

\begin{tabular}{|c|c|c|c|}
\hline & SEPSIS $(n=9)$ & COVID-19 $(n=9)$ & $p$ value \\
\hline Age & $57.67 \pm 4.97$ & $61.44 \pm 3.06$ & 0.53 \\
\hline Sex, \% male $(n)$ & $77.78(7)$ & $55.56(5)$ & 0.32 \\
\hline $\begin{array}{l}\text { SARS-CoV-2 infection confirmed by } \\
\text { PCR: } \%(n)\end{array}$ & $0(0)$ & $100(9)$ & $*<0.0001$ \\
\hline Intubation: \% ( $n)$ & $77.78(7)$ & $100(9)$ & 0.13 \\
\hline VV- or VA-ECMO: \% (n) & $11.11(1)$ & $55.56(5)$ & $* 0.04$ \\
\hline BMI & $24.98 \pm 2.02$ & $33.28 \pm 2.66$ & $* 0.02$ \\
\hline Diabetes, \% (n) & $22.22(2)$ & $22.22(2)$ & $>0.99$ \\
\hline Arterial Hypertension, \% ( $n)$ & $33.33(3)$ & $66.67(6)$ & 0.16 \\
\hline Coronary artery disease, $\%(n)$ & $11.11(1)$ & $22.22(2)$ & 0.53 \\
\hline Heart failure, \% $(n)$ & $22.22(2)$ & $11.11(1)$ & 0.53 \\
\hline End-stage renal disease, $\%(n)$ & $22.22(2)$ & $88.89(8)$ & $* 0.004$ \\
\hline Ferritin $^{\mathrm{a}}$ & $2747 \pm 2162(n=6)$ & $7455 \pm 4305(n=7)$ & 0.23 \\
\hline Serum iron $^{\mathrm{a}}$ & $125.8 \pm 57.43(n=4)$ & $26(n=1)$ & 0.49 \\
\hline Hemoglobin & $8.96 \pm 0.76$ & $10.08 \pm 1.22$ & 0.43 \\
\hline
\end{tabular}

$P C R$ polymerase chain reaction, $v v$ veno-venous, $v a$ veno-arterial, ECMO extracorporeal membrane oxygenation

${ }^{a}$ Number of values available differs from group number

$* p$ value $<0.05$ assay. Proteins were denaturated for $30 \mathrm{~min}$ at $37^{\circ} \mathrm{C}$ in $2 \%$ $\beta$-mercaptoethanol. Proteins were separated on $8 \%$ sodium dodecyl sulfate polyacrylamide gels, then transferred to a nitrocellulose membrane and incubated with the primary antibodies in 5\% milk in TBS-T at $4{ }^{\circ} \mathrm{C}$ overnight: antiFTH (ab65080, abcam, 1:1000), anti-FTL (ab69090, abcam, 1:1000) and as housekeeper anti-GAPDH (G8795, SigmaAldrich, 1:10,000). Secondary antibodies were HRP-conjugated donkey anti-rabbit and sheep anti-mouse IgG (NA934 and NA931, GE Healthcare, 1:10,000) that were incubated for $1 \mathrm{~h}$ at room temperature. For chemiluminescent detection, WesternBright ${ }^{\mathrm{TM}}$ ECL HRP substrate (K-12045-D50, advansta) was used. Protein expression was normalized to GAPDH expression using ImageJ to determine mean densitometry.

In addition, RNA was extracted from left ventricular tissue using trichloromethane-chloroform solution and isopropanol solution. RNA was purified using the RNeasy Plus Mini Kit (Qiagen) and transcribed to cDNA. Quantitative analysis was performed using the respective primer (FTH: Hs01694011_s1, FTL: Hs00830226_gH, GPX4: Hs00989766_g1, HMOX-1: Hs01110250_m1, Applied Biosystems) on a TaqMan apparatus (Applied Biosystems), and expression was normalized to $\beta$-actin (Hs00357333_g1, Applied Biosystems).

For detection of 4-HNE, a marker of oxidative stress, paraffin embedded hearts were cut in $4 \mu \mathrm{m}$ thick sections and fixed on slides. Before staining, sections were deparaffinized and rehydrated $(2 \times 10 \mathrm{~min}$ in xylol, $2 \times 5 \mathrm{~min}$ in $100 \%$
EtOH, $2 \times 5$ min in $96 \% \mathrm{EtOH}, 2 \times 5$ min in $70 \% \mathrm{EtOH}$, $5 \mathrm{~min}$ in PBS). Antigen retrieval was performed by $30 \mathrm{~s}$ boiling at $121{ }^{\circ} \mathrm{C}$ in Antigen Unmasking Solution (H-3300-250, Vector Laboratories, 1:100 in $\mathrm{dH}_{2} \mathrm{O}$ ). After blocking with SuperBlock ${ }^{\mathrm{TM}}$ Blocking Buffer (37515, Thermo Fisher Scientific) for $30 \mathrm{~min}$, heart sections were stained with Wheat Germ Agglutinin (WGA), Alexa Fluor ${ }^{\mathrm{TM}} 594$ Conjugate (W11262, Thermo Fisher Scientific, 1:300 in PBS, marker for cell membrane) and Anti-4 Hydroxynonenal antibody (4-HNE) (ab48506, abcam, 1:200 in PBS) antibody overnight $\left(4{ }^{\circ} \mathrm{C}\right)$. Donkey anti-mouse IgG Alexa Fluor ${ }^{\mathrm{TM}} 568$ (A10037, Invitrogen, 1:500 in PBS) was used as secondary antibody for 4-HNE ( $1 \mathrm{~h}$ at room temperature). Staining with DAPI (33342, Molecular Probes, 1:50,000 in PBS, 2 min) was done to visualize the nuclei. To quench autofluorescence, sections were incubated with Vector ${ }^{\circledR}$ TrueVIEW $^{\circledR}$ Autofluorescence Quenching Kit (SP-8400-15, Vector Laboratories) for $2 \mathrm{~min}$. For analysis, 40 images per section were recorded with a Zeiss Observer.Z1 microscopy (20x, Carl Zeiss Microscopy $\mathrm{GmbH}$ ) and percent of 4-HNE stained area of total area was calculated.

For statistical testing, normality was tested using the Kolmogorov-Smirnov test. For data with normal distribution, a Student's $t$ test, one-way ANOVA with Šídák's multiple comparisons test (for equal SD) or Brown-Forsythe/Welch ANOVA test with two-stage linear step-up procedure of Benjamini, Krieger and Yekutieli (no equal SD) was performed. For data, for which normality could not be assumed, Mann-Whitney test or Kruskal-Wallis 
with Dunn's multiple comparisons test was performed. For correlation analysis, correlation was tested using linear regression. The significance level was taken to 5\% (twosided $p$ ). Data are presented as mean $\pm \mathrm{SEM}$. Analyses and graphs were generated using GraphPad Prism 9 Statistical Software (GraphPad, San Diego, CA, USA).

Iron concentration in left ventricular myocardium of patients who had died due to COVID-19 was significantly elevated compared to tissue from the NF (nonfailing) group (Fig. 1A, NF: $n=6$, COVID-19: $n=7$ ). To determine the cause of increased iron accumulation in the heart of COVID-19 patients, we analyzed the expression of genes involved in iron metabolism.

Intracellular iron accumulation may be accompanied by decreased iron storage capacity, which can be determined by the ratio of heavy chain ferritin (FTH) to light chain ferritin (FTL). Because FTH differs from FTL in its ferroxidase activity, a decreased FTH:FTL ratio results in decreased intracellular storage capacity as well as an increased labile iron pool (LIP) size, which increases oxidative stress. Protein
Fig. 1 Iron metabolism in left ventricular (LV) myocardium of deceased COVID-19 patients. A In the LV myocardium of deceased COVID-19 patients, iron concentration is significantly increased compared to the non-failing group (NF; $p=0.004, t$ test, $n=6-7)$. B In LV myocardium of deceased COVID-19 patients, protein expression of ferritin heavy chain (FTH) to ferritin light chain (FTL) is significantly decreased compared to NF group ( $p=0.005$, Mann-Whitney test, $n=6-7)$. C In LV myocardium of deceased COVID-19 patients, ratio of ferritin heavy chain (FTH) to ferritin light chain (FTL) mRNA is significantly decreased compared to $\mathrm{NF}(p=0.007)$ and SEPSIS group ( $p=0.03$, Kruskal-Wallis test, $n=6-7)$. D In LV myocardium of deceased COVID-19 patients, glutathione peroxidase 4 (GPX4) is decreased compared to NF $(p=0.03)$ and SEPSIS group $(p=0.01$, BrownForsythe/Welch ANOVA test, $n=6-7)$. E In LV myocardium of deceased COVID-19 patients, heme oxygenase-1 (HMOX-

$1)$ is significantly increased compared to NF $(p=0.01)$ and SEPSIS group $(p=0.008$, oneway ANOVA test, $n=6-7)$. F In LV myocardium sections of deceased COVID-19 patients, 4-HNE staining, a marker for oxidative stress, is significantly enhanced compared to SEPSIS group ( $p=0.001, t$ test, $n=8$ each) (4-HNE: green, WGA: red, DAPI: blue). G 4-HNE staining and troponin levels are significantly correlated in COVID-19 patients ( $p=0.03$, $\mathrm{R}^{2}=0.57, n=8$, linear regression)
A

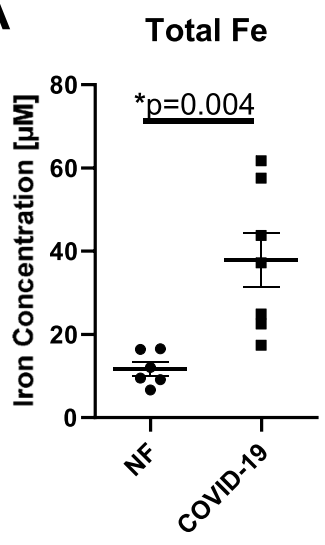

C
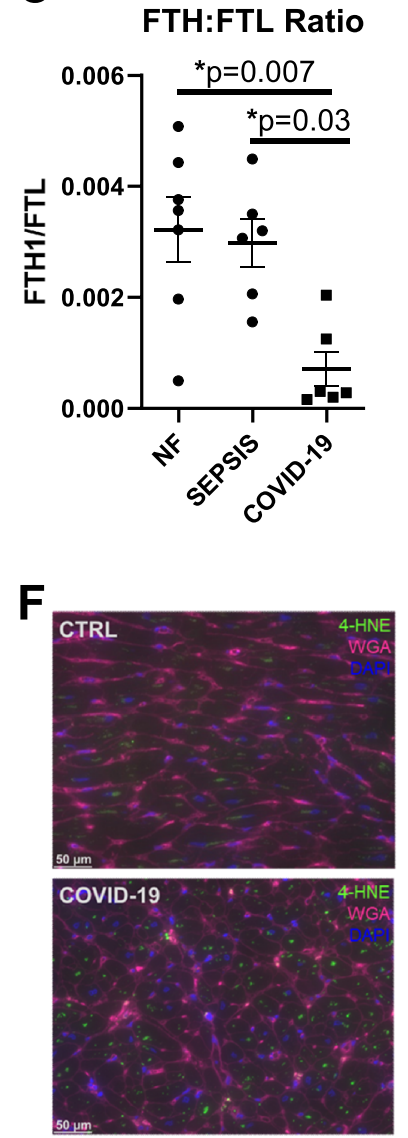

B

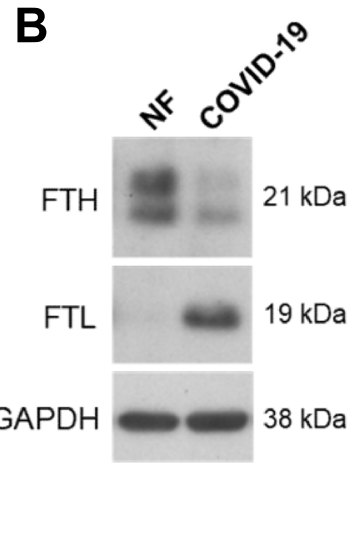

D
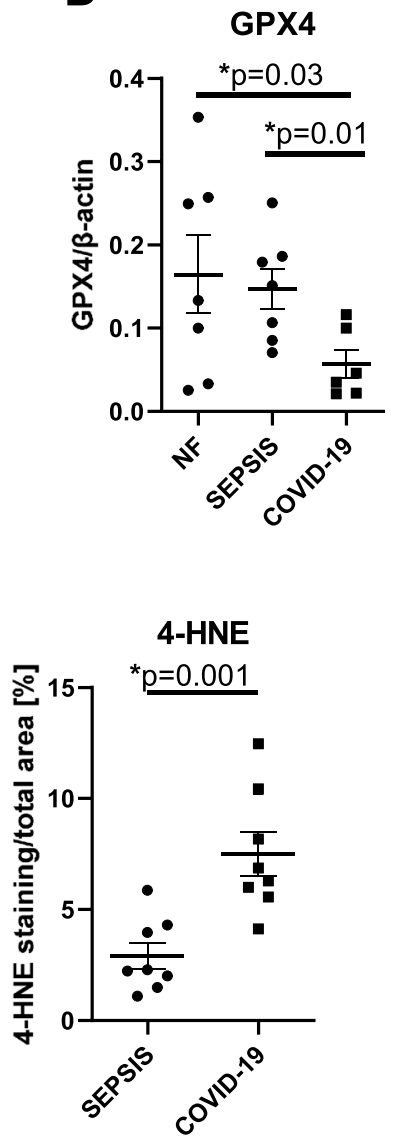

FTH:FTL Ratio

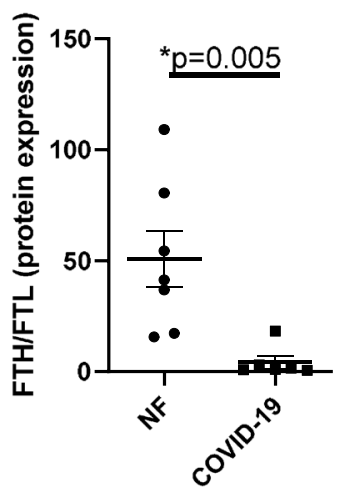

E

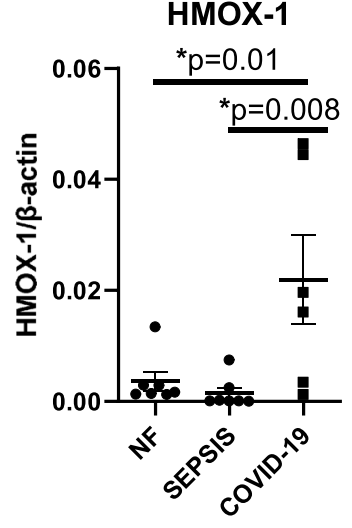

G

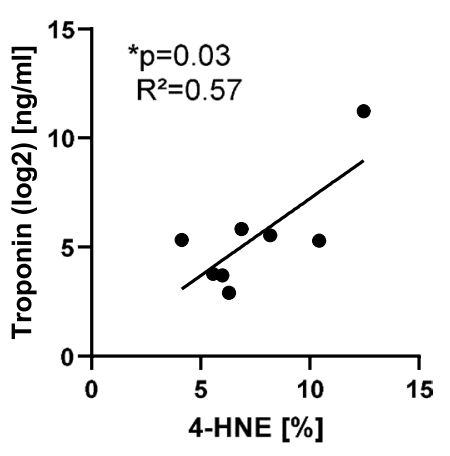


expression of FTH:FTL ratio was significantly decreased in patients with COVID-19 compared to NF group (Fig. 1B, NF: $n=7$, COVID-19: $n=6$ ). It could be argued, that ferritin expression might also be altered in patients with "classic" i.e. bacterial septic shock. However, the ratio of FTH to FTL gene expression in left ventricular myocardium of COVID19 patients was significantly decreased compared with tissues from the NF group, but also compared with patients who had died of sepsis (SEPSIS) (Fig. 1C, NF: $n=7$, SEPSIS: $n=6$, COVID-19: $n=6$ ).

Similarly, glutathione peroxidase 4 (GPX4), an enzyme converting reduced glutathione (GSH) to oxidized glutathione (GSSG), was decreased in patients with COVID19 compared with the NF and SEPSIS groups (Fig. 1D, NF: $n=7$, SEPSIS: $n=7$, COVID-19: $n=6$ ), which mechanistically should further increase the labile iron pool (LIP).

Moreover, the LIP can be enhanced by excessive activation of heme oxygenase-1 (HMOX-1) (non-canonical ferroptosis pathway). Heme oxygenase catalyzes the degradation of heme to $\mathrm{CO}$, biliverdin and free iron. In this study, HMOX-1 was significantly upregulated in patients who died from COVID-19 compared with both the NF and SEPSIS groups (Fig. 1E, NF: $n=7$, SEPSIS: $n=7$, COVID19: $n=6$ ), suggesting that both canonical and non-canonical ferroptosis pathways are involved in COVID-19 associated cell death. The increased LIP can lead to an excessive induction of reactive oxygen species (ROS) in the myocardium of these patients.

To test whether increased ROS-production leads to oxidative damage of the cells, we used 4-HNE (4-Hydroxynonenal) staining as a sensitive marker for oxidative damage and lipid peroxidation. The percentage area of 4-HNE staining was significantly increased in left ventricular sections from patients with COVID-19 compared with sections from patients in the SEPSIS group (Fig. 1F, SEPSIS: $n=8$, COVID-19: $n=8$ ). To test whether oxidative stress following myocardial iron overload could lead to myocardial damage in COVID-19 patients, we performed a correlation analysis between the percentage area of 4-HNE staining and troponin before death of the COVID-19 patients. Interestingly, our analysis showed a significant correlation of 4-HNE staining and troponin levels (Fig. 1G, COVID-19: $n=8$ ), which would be consistent with ferroptosis-induced myocardial injury in severe COVID-19.

The current study demonstrates that iron overload occurs in the myocardium during severe disease courses of COVID19. Intracellular iron accumulation is accompanied by decreased iron storage capacity (FTH:FTL ratio), inhibition of detoxifying enzymes (GPX4) as well as activation of the ferroptosis pathway (HMOX-1), leading to increased myocardial oxidative stress with subsequent myocardial damage (Fig. 2). Notably, our data suggest a mechanistic difference in iron metabolism between severe COVID-19 disease and

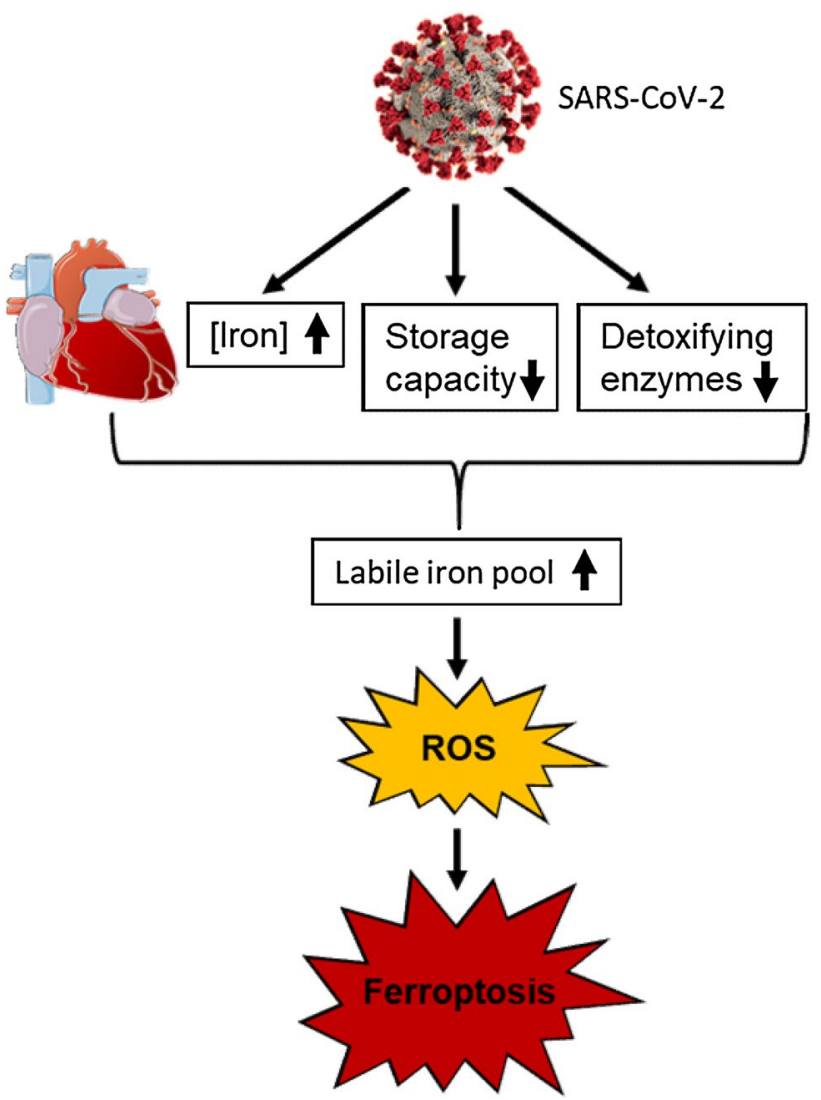

Fig. 2 Graphical overview. In myocardium, SARS-CoV-2 infection increases iron concentration, decreases intracellular iron storage capacity and inhibits detoxifying enzymes, leading to excessive increase in the labile iron pool (LIP). As a result, reactive oxygen species (ROS) formation and lipid peroxidation occur, leading to ferroptosis. (SARS-CoV-2 figure by CDC/Alissa Eckert, MS; Dan Higgins, MAMS)

bacterial sepsis. However, it is unknown whether impaired iron homeostasis predisposes to severe COVID-19 or is a direct effect of the disease.

Since iron is also important for replication of viruses including coronaviruses, direct manipulation of iron metabolism by SARS-CoV-2 and dysregulated iron metabolism as a consequence of viral infection seems possible. This could also explain the heterogeneous clinical symptoms and multiple organ involvement in COVID-19, as ferroptosis has been shown to be involved in neurological diseases, renal damage, the circulatory system, and even immune system regulation [7].

Dysregulation of iron metabolism is also implicated in other diseases, for example, type 2 diabetes or obesity [8]. At the same time, patients with these diseases have the highest risk of severe COVID-19, suggesting a possible link between severe COVID-19 and dysregulated iron metabolism.

Therefore, iron chelators, such as deferoxamine, deferiprone and deferasirox, may be a promising therapeutic 
option for the treatment of severe COVID-19 courses. However, the results of our current study should be extended to other organs in the near future and, because of the small number of patients included in this study, validated in larger patient cohorts. It would be also of interest to study subcellular iron distribution. Since iron serves as a catalyst for the chemical reaction, very small amounts of free iron would be sufficient to trigger ferroptosis $[9,10]$, even below detection limit of conventional histochemical techniques. Because we used only cardiac tissue samples from deceased patients, interpretation of our results for the living organism, especially for patients with asymptomatic or moderate COVID19 disease, may be difficult. However, it should be noted that 4-HNE staining correlated significantly with cardiac troponin levels in COVID-19 patients, suggesting that relevant ferroptosis may be present in severe COVID-19 disease. Therefore, the onset and progression of dysregulated iron metabolism during COVID-19 disease should be investigated in further studies.

In conclusion, this study is the first to show a direct association between intracellular iron accumulation, ferroptosis and SARS-CoV-2 infection in the heart during lethal severe disease courses of COVID-19.

Acknowledgements We would like to acknowledge the expertise technical assistance of Gabriela Pietrzyk and Sebastian Benkhoff.

Author contributions JM and MJB designed and wrote the manuscript, acquired the data, performed statistical analysis and are responsible for the integrity of the manuscript. KE and ME acquired the human tissue samples and revised the manuscript for critical intellectual content. $\mathrm{JH}, \mathrm{SW}, \mathrm{SN}, \mathrm{SS}, \mathrm{CJ}$, and LSM revised the manuscript for critical intellectual content.

Funding Open Access funding enabled and organized by Projekt DEAL. MJB is supported by the German Heart Foundation/German Foundation of Heart Research-F/50/20. JM is funded by the German Cardiac Society Clinician Scientist program and by the Deutsche Forschungsgemeinschaft (DFG, German Research Foundation) grant MU 4555/2-1 (project number 455425596). SN is funded by DFG grant NE 2159/3-1 (project number 455425596). LSM is funded by DFG grant MA1982/5-1. SW and LSM are funded by SFB 1350 TPA6 and the University of Regensburg ReForM C programme. SW is funded by DFG grants WA 2539/4-1, 5-1, 7-1, and 8-1.

Availability of data and materials Original data can be made available in a blinded manner upon reasonable request.

Code availability Not applicable.

\section{Declarations}

Conflict of interest No conflict of interest declared.

Ethics approval All investigations conform to the Declaration of Helsinki.
Consent to participate and for publication Informed consent for autopsies involving scientific investigations had been obtained from relatives of all deceased patients, which was performed in accordance with institutional and ethics guidelines.

Open Access This article is licensed under a Creative Commons Attribution 4.0 International License, which permits use, sharing, adaptation, distribution and reproduction in any medium or format, as long as you give appropriate credit to the original author(s) and the source, provide a link to the Creative Commons licence, and indicate if changes were made. The images or other third party material in this article are included in the article's Creative Commons licence, unless indicated otherwise in a credit line to the material. If material is not included in the article's Creative Commons licence and your intended use is not permitted by statutory regulation or exceeds the permitted use, you will need to obtain permission directly from the copyright holder. To view a copy of this licence, visit http://creativecommons.org/licenses/by/4.0/.

\section{References}

1. Sattar Y, Ullah W, Rauf H, Virk HUH, Yadav S, Chowdhury M, Connerney M, Mamtani S, Pahuja M, Patel RD, Mir T, Almas T, Moussa Pacha H, Chadi AM. COVID-19 cardiovascular epidemiology, cellular pathogenesis, clinical manifestations and management. Int J Cardiol Heart Vasc. 2020;29: 100589. https://doi.org/ 10.1016/j.ijcha.2020.100589.

2. Sonnweber T, Boehm A, Sahanic S, Pizzini A, Aichner M, Sonnweber B, Kurz K, Koppelstätter S, Haschka D, Petzer V, Hilbe R, Theurl M, Lehner D, Nairz M, Puchner B, Luger A, Schwabl C, Bellmann-Weiler R, Wöll E, Widmann G, Tancevski I, Löffler-Ragg J, Weiss G. Persisting alterations of iron homeostasis in COVID-19 are associated with non-resolving lung pathologies and poor patients' performance: a prospective observational cohort study. Respir Res. 2020;21:276. https://doi.org/10.1186/ s12931-020-01546-2.

3. Bellmann-Weiler R, Lanser L, Barket R, Rangger L, Schapfl A, Schaber M, Fritsche G, Wöll E, Weiss G. Prevalence and predictive value of anemia and dysregulated iron homeostasis in patients with COVID-19 infection. J Clin Med. 2020;9:2429. https://doi. org/10.3390/jcm 9082429 .

4. Hippchen T, Altamura S, Muckenthaler MU, Merle U. Hypoferremia is associated with increased hospitalization and oxygen demand in COVID-19 patients. Hemasphere. 2020;4: e492. https://doi.org/10.1097/HS9.0000000000000492.

5. Chiang SK, Chen SE, Chang LC. A dual role of heme oxygenase-1 in cancer cells. Int J Mol Sci. 2018;20:39. https://doi.org/10.3390/ ijms20010039.

6. Mustroph J, Sag CM, Bähr F, Schmidtmann AL, Gupta SN, Dietz A, Islam MMT, Lücht C, Beuthner BE, Pabel S, Baier MJ, ElArmouche A, Sossalla S, Anderson ME, Möllmann J, Lehrke M, Marx N, Mohler PJ, Bers DM, Unsöld B, He T, Dewenter M, Backs J, Maier LS, Wagner S. Loss of CASK accelerates heart failure development. Circ Res. 2021;128:1139-55. https://doi.org/ 10.1161/CIRCRESAHA.120.318170.

7. Tang D, Chen X, Kang R, Kroemer G. Ferroptosis: molecular mechanisms and health implications. Cell Res. 2021;31:107-25. https://doi.org/10.1038/s41422-020-00441-1 (Epub 2020 Dec 2).

8. Ravingerová T, Kindernay L, Barteková M, Ferko M, Adameová A, Zohdi V, Bernátová I, Ferenczyová K, Lazou A. The molecular mechanisms of iron metabolism and its role in cardiac dysfunction and cardioprotection. Int J Mol Sci. 2020;21:7889. https://doi.org/ 10.3390/ijms21217889. 
9. Fang X, Wang H, Han D, Xie E, Yang X, Wei J, Gu S, Gao F, Zhu N, Yin X, Cheng Q, Zhang P, Dai W, Chen J, Yang F, Yang HT, Linkermann A, Gu W, Min J, Wang F. Ferroptosis as a target for protection against cardiomyopathy. Proc Natl Acad Sci USA. 2019;116:2672-80. https://doi.org/10.1073/pnas.1821022116.
10. Toyokuni S, Ito F, Yamashita K, Okazaki Y, Akatsuka S. Iron and thiol redox signaling in cancer: an exquisite balance to escape ferroptosis. Free Radic Biol Med. 2017;108:610-26. https://doi.org/ 10.1016/j.freeradbiomed.2017.04.024. 